\title{
PROSPECTS FOR FUTURE ASTROMETRIC MISSIONS
}

\author{
P.K. SEIDELMANN \\ U.S. Naval Observatory
}

\begin{abstract}
Hipparcos and the Hubble Space Telescope have demonstrated the astrometric capabilities in space. SIM and GAIA are being studied for future missions. There have been many proposals for new astrometry missions from many different countries, but most of them have not been funded.

The best possibility for a mission within the next five years would be through a collaborative effort for a small, fast, cheap spacecraft which could be a precursor for future, larger, more accurate missions, which are under study.
\end{abstract}

\section{Introduction}

In 1997 there will be available the Hipparcos Astrometric Satellite star catalog of 120,000 stars with accuracies of 1 milliarcsecond (mas) and proper motions of 1.1 mas year $^{-1}$ for stars brighter than 9 th magnitude. In addition, the Tycho Catalog will include one million stars with accuracies of 30 mas. The Hubble Space Telescope can do only a limited amount of astrometry due to the competition for observing time.

Ground-based astrometric, optical observations over wide angles will be made at mas accuracies with optical interferometers, such as the Navy Prototype Optical Interferometer, for several thousand stars. CCD observations of small fields can be made with $30-50$ mas relative accuracies.

There are many proposals for future astrometric missions. They can be divided into scanning and pointed missions. The scanning missions, like Hipparcos, can observe many stars repeatedly and achieve good accuracy. The pointed missions measure relative positions more accurately, but cannot make as many observations and cannot observe as many stars. The technological improvements are based on the use of CCD detectors, optical 
interferometers, metrology systems, and increased data rates. The Space Interferometry Mission (SIM) in the U.S. and the Global Astrometric Interferometer for Astrophysics (GAIA) in Europe have some financial support for studies.

\section{Why Future Astrometric Space Missions?}

The real prospects for future astrometric missions must be tied to the reasons for such missions. The observables are positions, proper motions, parallaxes, photometry and images. New missions can achieve more accuracy and more stars. There are the traditional astrometric applications of navigation, guidance, and reference frames. In addition, space activities have added space surveillance and autonomous space navigation as applications for accurate astrometric data. However, this probably will not justify a future astrometric mission. So consider the purpose in terms of NASA's four themes, where astrometry has a fundamental scientific role:

\subsection{STRUCTURE AND EVOLUTION OF THE UNIVERSE}

- Calibrate the cosmological distance scale by measuring absolute parallaxes of Cepheids and RR Lyrae.

- Detemine positions, proper motions, and absolute parallaxes to $10 \%$ accuracy or better (as well as apparent magnitudes and spectral energy distributions) of a complete sample of stars brighter than 14th magnitude within 2.5 kiloparsecs of the Sun.

- Calibrate the absolute luminosities of solar neighborhood stars, including population I and II stars, enabling studies of stellar evolution.

- Determine transverse velocities of a complete sample of stars within 2 $\mathrm{kpc}$ of the Sun to 14th magnitude. From this, the mass distribution and gravitational surface mass density in the disk near the Sun can be determined. This relates directly to the dark matter implied by dynamical studies of globular clusters and rotation of galaxies (the "Oort problem").

- Detect astrometric perturbations of lensed sources during a microlensing event, and directly image a MACHO object shortly after a lensing event.

- Determine parallaxes and relative positions of binaries to determine masses, including unusual systems such as those containing white dwarfs and black holes, by analysis of positions of photocenter emission and use of multiple colors.

- Calibrate the distances to open star clusters and determine the absolute color magnitude diagrams of newly formed star clusters. 
- Determine accurate reference frames which will ensure identification of sources in radio, optical, infrared and X-ray wavelengths.

\subsection{SEARCH FOR ORIGINS AND PLANETARY SYSTEMS.}

- Identify candidate stars for brown dwarfs and planets from inconsistent proper motion values.

- Detect astrometrically planets and brown dwarfs from non-linear proper motions.

- Image brown dwarfs and planets directly using interferometric nulling.

- Image the scattered light from the disks and exo-zodiacal dust surrounding young stars and main sequence stars like Beta Pictoris using interferometric nulling.

- Calibrate the cosmological distance scale which is critical to the origins of the universe question.

\subsection{SOLAR SYSTEM EXPLORATION.}

- Determine the accurate relationships between the radio, optical and dynamical reference frames which are necessary for solar system exploration.

- Make accurate positional observations of asteroids which will contribute to determination of asteroid masses.

- Make accurate observations of small bodies to improve the ephemerides for mission objectives.

- Make accurate observations of positions of near Earth objects which will improve knowledge of the motions of these objects.

\subsection{SUN EARTH CONNECTION.}

- Image other Suns and determine their radius, mass, luminosity and distance.

\section{Possible Future Astrometric Missions}

At this time two missions seem to be the most likely for future launch and are being actively studied. The Space Interferometer Mission (SIM) (OSI 1996) is a U.S. spacecraft with pointed Michelson interferometers of variable baselines and a nulling interferometer backend. It can observe about 5000 stars down to 20th magnitude to an accuracy of 0.004 mas. Plans are to launch SIM by 2005. It is a precursor for the ExNPS mission and will test technology for that future mission. 
The Global Astrometric Interferometry for Astrophysics (GAIA) (Lindegren and Perryman 1995) is a European Space Agency study for a stack of three scanning Fizeau interferometers. It can observe 50,000,000 stars at an accuracy of 0.010 mas brighter than 15 th magnitude. It is currently competing for a cornerstone mission with a possible launch date of 2015 .

TABLE 1. Small scanning astrometric missions.

\begin{tabular}{llll}
\hline & FAME & LIGHT & DIVA \\
\hline Purpose & Astrometry & Galactic & Astrometry \\
& Photometry & Halo Tracer & Photometry \\
Technique & Fixed Angle & Scanning Fizeau & $1 / 10$ size \\
& Scanning & Interferometer & Gaia \\
Num. of Stars & $10,000,000$ & $10,000,000$ & 100,000 \\
Accuracy & 0.05 mas & 0.1 mas & 0.8 mas \\
Mag. Limit & 15 & 15 & 10.5 \\
Launch Date & 2001 & 2007 & 2005 \\
Status & Proposal & Proposal & Proposal \\
\hline
\end{tabular}

There are currently three proposals (Table 1) for a scanning instrument similar to Hipparcos, but using CCD detectors and higher data rates, so that more stars and accuracy can be achieved: FAME from USA (Seidelmann et al. 1995), LIGHT from Japan (Yoshizawa et al. 1997), and DIVA from Germany (Bastian et al. 1997). One such mission would fill the needs for a large all sky, very accurate star catalog down to about 15th magnitude. In addition to excellent science, such as calibrating the distance scale, this project would provide both a second epoch for Hipparcos and an independent set of short-time-period proper motions. The comparison between the sets of short-period proper motions and long-period proper motions should identify discrepancies and thus most likely candidates for planetary systems and brown dwarfs. This type of project is currently the most needed, unfunded astrometric spacecraft.

Two pointed spacecraft have been proposed (Table 2), POINTS (Chandler and Reasenberg 1990) and a scaled down POINTS called Newcomb (Johnston et al. 1995). These have been supplemented by the SIM project. A number of Russian spacecraft have been proposed in the last decade. The three most actively being discussed at the present time are AIST or Struve (Yershov et al. 1995; Chubey et al. 1995; Kopylov et al. 1995), Lomonosov (Nesterov et al. 1990) and Zenith (Table 3). 
TABLE 2. Pointed astrometric missions.

\begin{tabular}{lll}
\hline & NEWCOMB & POINTS \\
\hline Purpose & Astrometry & Astrometry \\
Technique & Stacked, small Michelson & Rigid Michelson \\
& Interferometer & Interferometer \\
Num. of Stars & 3,000 & 5,000 \\
Accuracy & 0.1 mas & 0.001 mas \\
Mag. Limit & 15 & 12 \\
Launch Date & $?$ & $?$ \\
Status & Unfunded & Unfunded \\
\hline
\end{tabular}

TABLE 3. Russian astrometric missions.

\begin{tabular}{llll}
\hline & AIST/STRUVE & LOMONOSOV & ZENITH \\
\hline Purpose & Astrometry & Astrmetry & Astrometry \\
& Photometry & Photometry & \\
Technique & 2 Schmidt & 1 m Mirror & Michelson \\
& telescopes, & $90^{\circ}$ separation, & Interferometer, \\
& Fixed angle & rotating & pointing \\
& scanning & & \\
Num. of Stars & 500,000 & 400,000 & 3,000 \\
Accuracy & 0.3 mas & $2-10$ mas & 0.1 mas \\
Mag. Limit & 18 & 10 & 12 \\
Launch Date & 2001 & 2003 & $?$ \\
Status & Study & Study & $?$ \\
\hline
\end{tabular}

There are also discussions about observations from the Moon (Mission , the Moon 1992). These proposals try to take advantage of the large rface, absence of interference, distance to Earth, or lunar characteristics able 4). All of these ideas appear to be far in the future.

\section{Conclusion}

he Hipparcos satellite has proven to be a great success and demonstrated e capability to do astrometry in space. Since its design, there have been gnificant technical developments that make it possible to build a satel- 
TABLE 4. Proposed lunar based observations.

\begin{tabular}{ll}
\hline TECHNIQUE & PURPOSE \\
\hline VLF Array of antennas & Extragalactic \\
Optical Interferometer & Small angel astrometry \\
Earth-Moon VLBI & Astrometry \\
Complex Optical Interferometer & Imaging \\
1 M Transit Telescope with CCDs & Macho detection \\
\hline
\end{tabular}

lite which can observe many more stars much more accurately. There is excellent scientific justification for future astrometric satellites.

As a result many satellites have been proposed for astrometric, photometric and imaging purposes. At this time the SIM and GAIA proposals appear to be the most likely to be launched. These two satellites complement each other in their capabilities and the differences in their launch dates. There appears to be good scientific justification for a scanning satellite with 50 microarcseconds accuracies for stars brighter than 9 magnitude launched in the first years of the twenty first century.

\section{References}

Bastian, U.; Hog, E.; Mandel, H.; Quirrenback, A.; Roser, S.; Schalinski, C.; Schilback, E.; Seifert, W.; Wanger, S.; and Wicenec, A. (1997) "DIVA, An Interferometric Minisatellite for Astrometry and Photometry" Astronomische Nachrichten (in preparation)

Chander, J.F. and Reasenberg, R. D. 1990, "POINTS: A Global Reference Frame Opportunity," Inertial Coordinate System on the Sky, J. H. Lieske and V. K. Abalakin eds, Kluwer Academic Publishers, Dordrecht, 217-228.

Chubey, M.S.; Paskkov, V.S.; Kopylov, I.M.; Kirian, T. R.; Nickiforov, V. V.; Markelov, S.V; Ryadchenko, V.P. (1995) "On the Registration System of the AIST-Project" Astronomical and Astrophysical Objectives of Sub-Milliarcsecond Optical Astrometry, E. Hog and P. K. Seidelmann eds, Kluwer Academic Publishers, Dordrecht, 323-326.

Eichhorn, H. (1974) "Astronomy of Star Positions" Frederick Ungar Publishing Co, New York

Hindsley, R. B. and Harrington, R.S. (1994) "The U.S. Naval Observatory Catalog of Positions of Infrared Stellar Sources" Astron.J. 107, 280-6

Hog, E. (1995) "A New Era of Global Astrometry II. a 10 Microarcsecond Mission" Astronomical and Astrophysical Objectives of Sub-Milliarcsecond Optical Astrometry, E. Hog and P. K. Seidelmann eds, Kluwer Academic Publishers, Dordrecht, 317-322.

Johnston, K. J.; Seidelmann, P.K.; Reasenberg, R. D.; Babcock, R.; Phillips, J.D. (1995) "Newcomb Astrometric Satellite" Astronomical and Astrophysical Objectives of SubMilliarsecond Optical Astrometry, E. Hog and P. K. Seidelmann eds, Kluwer Academic Publishers, Dordrecht, 331-334.

Kopylov, I.M.; Gorshanov, D.L.; Chubey, M.S (1995) "Photometry Facilities of the AIST Space Project" Astronomical and Astrophysical Objectives of Sub-Milliarcsecond Op- 
tical Astrometry, E. Hog and P. K. Seidelmann eds, Kluwer Academic Publishers, Dordrecht, 327-330.

Lindegren, L.; Perryman, M.A.C. (1995) "A Small Interferometer in space for Global Astrometry: The GAIA Concept" Astronomical and Astrophysical Objectives of Sub-Milliarcsecond Optical Astrometry, E. Hog and P. K. Seidelmann eds, Kluwer Academic Publishers, Dordrecht, 337-344.

"Mission to the Moon," European Space Agency, esa SP-1150, June 1992.

Nesterov, V. V. Ovchinnikov, A. A., Cherepashchuk, A. M. and Sheffer E. K. 1990 "The Lomonosov Project for Space Astrometry," Inertial Coordinate System on the Sky, J. H. Lieske and V. K. Abalakin eds, Kluwer Academic Publishers, Dordrecht, 355-360.

OSI, Orbiting Stellar Interferometer, NASA Science and Technology Review, March 1996, JPL.

Seidelmann, P. K.; Johnston, K. J.; Urban, S.; Germain, M.; Corbin, T.; Shao, M.; Yu, J.; Fanson, J.,; Rickard, L. J.; Weiler, K., and Davinic, N. (1995) "A Fizeau Optical Interferometer Astrometric Satellite" in Future Possibilities for Astrometry in Space, a Joint RGO-ESA Workshop, Cambridge UK 19-21 June 1995 ESA SP-379

Volonte, S. (1995) "Astronomy from a Lunar Base," Astronomical and Astrophysical Objectives of Sub-Milliarcsecond Optical Astrometry, E. Hog and P. K. Seidelmann eds, Kluwer Academic Publishers, Dordrecht, 347-350.

Yoshizawa, M.; Sato, K.; Nishikawa, J.; and Fukushima, T. (1997). Two Astrometric Projects: MIRA (Mitaka optical and infrared interferometer Array) and LIGHT (Light Interferometer Satellite for the Studies of Galactic Halo Tracers); Proceedings of IAU Colloquium 165, Wytrzyszczak, Lieskie, and Mignard eds, Kluwer Academic Publishers.

Yershov, V. N.; Chubey, M.S.; Il'in, A.E.; Kopylov, I.M.; Gorshavov, D.L.; Kanayev, I.I.; and Kirian, T.R. (1995) "Struve Space Astrometric System. Scientific Grounds of the Project," (in Russian).

"Future Possibilities for Astrometry in Space" A Joint RGO-ESA Workshop, Cambridge UK 19-21 June 1995 ESA SP-379 